\title{
Fiducial Points Detection using SVM LINEAR CLASSIFIERS
}

\author{
Luiz Eduardo S. e Silva ${ }^{1}$, Pedro Donadio de T. Júnior ${ }^{1}$, Kenny V. dos \\ Santos $^{2}$ and Waldir S. S. Junior ${ }^{3}$ \\ ${ }^{1}$ PPGEE/CETELI, Federal University of Amazonas, Manaus, Amazonas, Brazil \\ luiz.edu.sales@gmail.com, pedrodonadio7@gmail.com, \\ ${ }^{2}$ DEQ/PPGEE/CETELI, Federal University of Amazonas, \\ Manaus, Amazonas, Brazil \\ kennyvinente@ufam.edu.br \\ ${ }^{3}$ DTEC/PPGEE/CETELI, Federal University of Amazonas, \\ Manaus, Amazonas, Brazil \\ waldirjreufam.edu.br
}

\begin{abstract}
Currently, there is a growing interest from the scientific and/or industrial community in respect to methods that offer solutions to the problem of fiducial points detection in human faces. Some methods use the SVM for classification, but we observed that some formulations of optimization problems were not discussed. In this article, we propose to investigate the performance of mathematical formulation C-SVC when applied in fiducial point detection system. Futhermore, we explore new parameters for training the proposed system. The performance of the proposed system is evaluated in a fiducial points detection problem. The results demonstrate that the method is competitive.
\end{abstract}

\section{KEYWORDS}

Pattern Recognition, SVM, Fiducial Points \& Parameter Grid

\section{INTRODUCTION}

Currently, the interest of scientific community and industry in methods to solve fiducial point detection problem is increasing (salient points [1], for example, tip of the nose) in human faces [1].

Typically, recent approaches can be divided in two main categories: local and global. In local methods, the fiducial points are detected and processed individually and no additional information is used. The global methods are characterized by detecting more fiducial points using deformable models, less susceptible to pose and illumination variations than local methods.

The classifier design is the most important stage of a fiducial point detection (supervised). In this stage, several machine learning techniques can be used [1] e [2]. Particularly, some studies [3] use a classification method called Support Vector Machine [4]. The mathematical formulation of SVM is obtained by optimization problem with restrictions [5].

Dhinaharan Nagamalai et al. (Eds) : CSE, DBDM, CCNET, AIFL, SCOM, CICS, CSIP - 2014

pp. 23-31, 2014. (C) CS \& IT-CSCP 2014

DOI : $10.5121 /$ csit.2014.4403 
There are many recent papers about this theme. For example, the authors propose in [2] a face recognition subsystem framework that uses fiducial points detection. The detection of these points combines two techniques. The first uses Gabor filters coefficients for local detecting and the second uses a combination of human face anthropometric measurements. The system proposed in [8] explores the same problem. The authors use classifiers based on IPD (Inner Detector Product) correlation filters. In [1], the authors developed robust filters to investigate the same problem. These filters are designed using the principal components.

In this article, we study the performance of SVM mathematical formulation called C-SVC (Support Vector Classification) [5], for fiducial point detection. The main contribution of this paper is the investigation of parameters that have not been used before. For performance evaluation, the classifiers were designed based on C-SVC. The training algorithm was performed using eleven (11) fiducial points in a database composed of 503 images (base subset BioID [7]). The performance of the system with C-SVC classifier was compared with similar methods, two of them using discriminating and filtering and one using linear SVM [1]. The results demonstrate the proposed method has competitive performance, and, in some cases, the proposed method outperforms the others.

The remainder of this paper is organized as follows. In next section, we present a SVM description. The system proposed is shown in Section 3, which is emphasized in the design of classifiers, parameters optimization of different types of SVM and the chosen parameters, in training and testing, results, and comments are made about these results. Finally, conclusions are drawn in Section 4.

\section{SUPPORT VECTORS MACHINES (SVM)}

The SVM, proposed by Vapnik [4], is used to solve classification and regression problems. The SVM algorithm provides an optimal separating hyperplane with maximum margin. The SVM can be formulated to consider mislabelled samples. In this case, the technique is called soft margin [5]. The use of soft margin provides a generalization of the SVM method to deal with problems of separating classes. The mathematical formulation can be written as follows. First, consider the hyperplane:

$$
y_{i}\left(\mathbf{w}^{t} \mathbf{x}_{i}+b\right) \geqslant 1-\xi_{i}, \text { to } i=\{1, \ldots, n\} .
$$

where: $\xi_{i}$, is the non-negative slack variable, $y_{i}$ is the class labels, $n$ is the number of total class elements and the superscript ${ }^{t}$ is the transpose of the vector. To maximize the class-separating margin and obtain the optimal hyperplane, it is necessary to find a solution to the optimization problem given by:

$$
\begin{array}{ll}
\text { Minimize } & \frac{1}{2}\|\mathbf{w}\|^{2}+C\left(\sum_{i=1}^{n} \xi_{i}\right) \\
\text { Restrict to } & y_{i}\left(\mathbf{w}^{t} \mathbf{x}_{i}+b\right) \geqslant 1-\xi_{i} \\
& \text { to } i=\{1, \ldots, n\}
\end{array}
$$

In Equation (2), $\mathrm{C}$ represents the weight imposed on the soft margin. The implementation of this optimization problem is known as C-SVC [5]. There are others SVM optimization problems that differ in some aspects such as: the formulation of the optimization problem and the used parameters [5]. The performance depends on the parameters choice and the search for the best set of parameters is extremely important. In this work, we use classifiers C-SVC due to its linear structure. The linear SVM classifiers have low computational cost and therefore we can say that the categorization of patterns is fast. 


\section{Fiducial Point Detection System Using C-SVC Classifiers}

\subsection{Design of Classifiers C-SVC}

As mentioned in Section II, we can find several SVM formulation of the optimization problem. In our research, we employ the optimization problem denominated C-SVC to design classifiers for a fiducial points detection system. An important aspect is investigate which set of parameters produces the best performance of the C-SVC classifiers. In this work, we explore the following parameters:

- Parameter of regularization $C$ with values equal to $2^{n}$ with $n=\{-12,-9,-6,-3,0,3\}$;

- Maximum number of iterations to convergence: $10000 ; 18000 ; 26000 ; 34000 ; 42000$; 50000.

- Proportional weighting of the number of class elements (positive: negative): (1:1), (1:5), (2:10), (10:500), (5:1), (10:2) and (500:10).

The range of parameter values were obtained empirically by preliminary results. In these cases, we observe the range have a significant influence in the performance of the fiducial points detection system. An important point of view is that each parameter and its ranges represent one experiment which must be explored. This approach is called parameters grid [5]. In our research, we have an extensive grid with 252 parameter combinations. We use $\xi_{i}$ equal to 0.1 .

\subsection{Fiducial Points Detection System using C-SVC Classifiers}

The fiducial points detection system has two stages: training and testing. In both, all images are pre-processed using a common step. The pre-processing step is composed by two stages. Firstly, we apply the Viola-Jones face detection algorithm [11] in the image. After that, the face is scaled to $200 \times 200$, and then we perform illumination correction [12].

In the training stage (see Figure 1), first, the image is pre-processed. Next, we use a method to select an elliptical region of interest with high probability of containing the fiducial point. The mathematical formulation can be seen in [1]. We can view the elliptical region of interest in the block output of the Gaussian Priori Model (Figure 1).

Following, we choose the $B_{z}$ blocks, with dimensions $13 \times 13$, that define the positive and negative classes, where $\mathbf{z}$ represents the coordinates of the center of the block. We select a restricted number of negative blocks due to its high amount. This selection is done using an algorithm to generate random two-dimensional coordinates called Salt and Pepper [13]. The positive and negative ratio is 160/1. Next, we transform each block $B_{z}$ (array) in a vector $v\left(B_{z}\right)$ by concatenating the rows of the transposed matrix. Finally, we use the blocks of positive and negative classes to design the C-SVC classifier. It is important comment that all images used in the test system are different from the images used in the training system, i.e., the sets of training and testing images are mutually exclusive. 


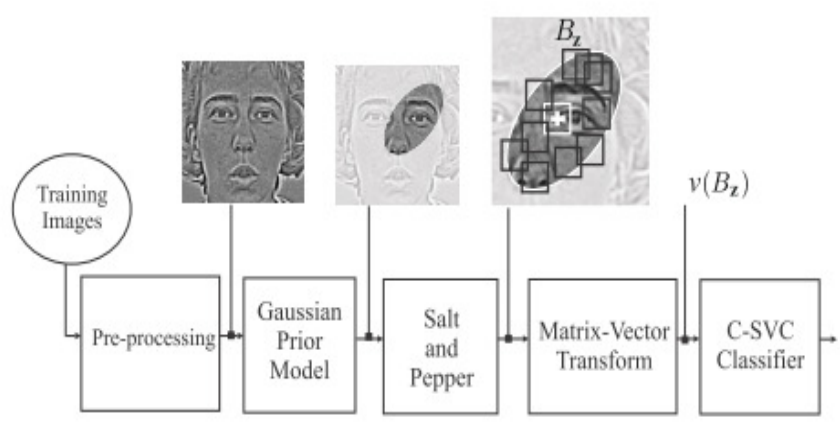

Figure 1. Block diagram for training the fiducial points detection system using C-SVC classifiers.

In the testing stage, shown in Figure 2, can be described as follows: first, we pre-processing the image. Next, we use the same elliptical region of interest determined in the training stage. Following, each block $\mathrm{B} \mathrm{z}$ is processed using a sliding window. The vector $v\left(B_{z}\right)$ is obtained through the transformation matrix $B_{z}$ in a vector (identically to the transformation performed in the training). Finally, we use the C-SVC classifier, found in the training, to label the vector $v\left(B_{z}\right)$ as positive or negative.

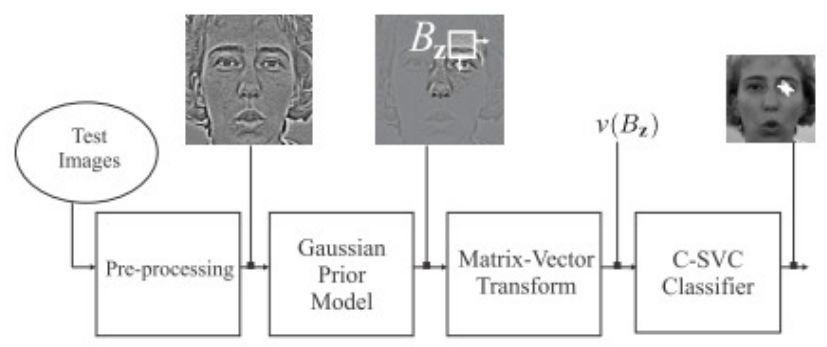

Figure 2. Block diagram for test the fiducial points detection system using C-SVC classifiers.

\subsection{Experiments}

The C-SVC classifiers of training and testing procedures (see Sections 3.1 and 3.2) were designed using OpenCV (Open Source Computer Library) [14]. OpenCV is written in C and C++ and can be operated in Windows, Linux and MAC OS. OpenCV was designed for computational efficiency and has specific mathematical functions and methods, for example, in signal processing and machine learning areas.

In order to evaluate the proposed method, we use 11 fiducial points as illustrated in Figure 3 . We use the system described in Section 3.2 for each fiducial point. Thus, we have 11 different fiducial points detection systems. The tests were made using cross-validation [15] with 7 partitions (value generally found in the literature [16]). In each experiment, we used two different sets for training and test. That total numbers of images in the database, we used $6 / 7$ for verification and training parameters and $1 / 7$ to validate the performance of the algorithm. 


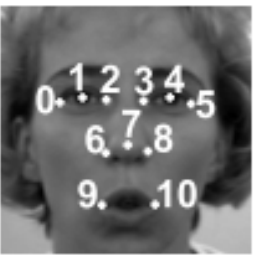

Figure 3. Fiducial points and a human face considered in the experiments.

The experiments were performed by cluster computer with 30 cores, 4GB RAM memory and $2.26 \mathrm{GHz}$ processor. In a brief description, BioID database [7] have 1521 images with frontal human faces in grey scale from 23 different people. The image resolution is $384 x 286$ pixels. The faces have change of scale (for instance, some are close to the camera and), lighting, and small rotations. Some people wear glasses, others have beard and/or moustache. The images in this database have manual labels for 20 fiducial points. In this work, we use a BioID subset with 503 images. We used only the frontal face images whose individuals do not wear glasses and do not have moustaches or beards. The faces have scale and lighting variations, and small rotations. Were performed 8316 experiments for C-SVC classifier parameter design and 11 fiducial points.

\subsection{Results}

The performance of classifier is assessed computing the True Positive (TP) and False Positive rates. We consider a candidate as a true positive when the distance between automatic and manual annotations is smaller than $10 \%$ of face intraocular distance (distance between the pupils).

In Figures 4, 5, 6, 7 and 8 are shown the simulations results of the points 04, 05, 07, 08 and 10. The graphics present the average TP, FP and standard deviation rates of all folds of all parameters grid. Each number in grid axis represents one parameter combination of set described in Section 3.1. To assess in order to evaluate the graphs, we consider the best performing regions those with higher TP and smaller FP rates jointly. Note that, for all graphs, the 84 first combinations are best performance of the parameters grid.
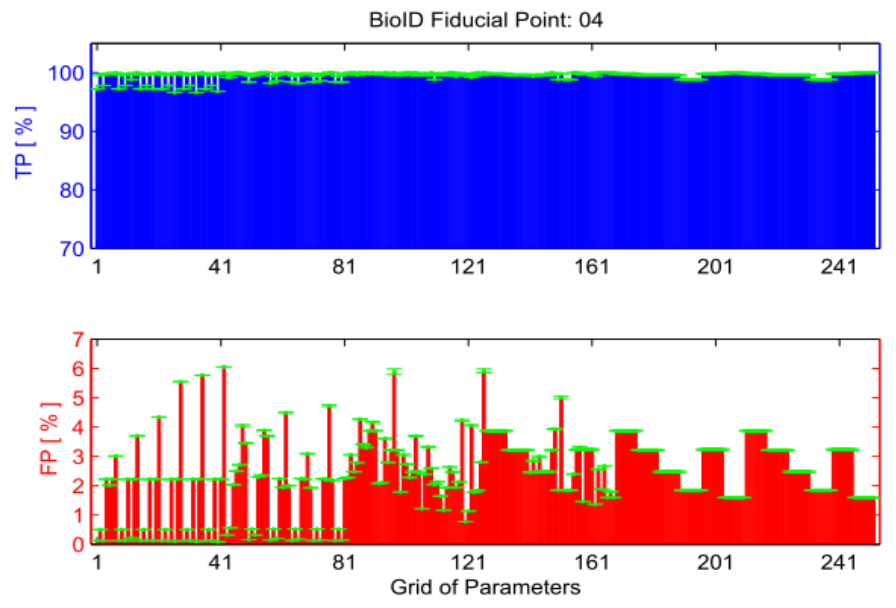

Figure 4. Grid of parameters for the point 04 (left eye pupil) from BioID dataset. 

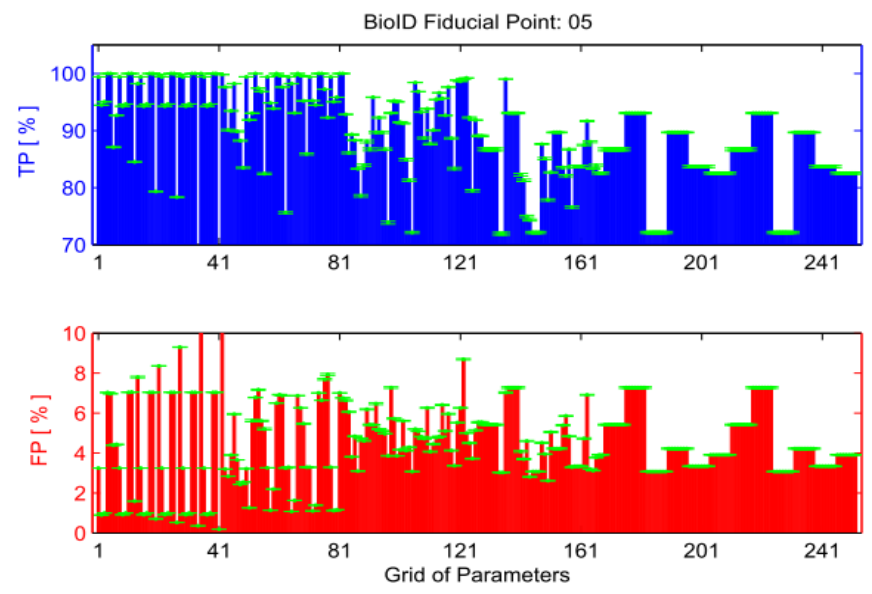

Figure 5. Grid of parameters for the point 05 (outer corner of left eye) from BioID dataset.
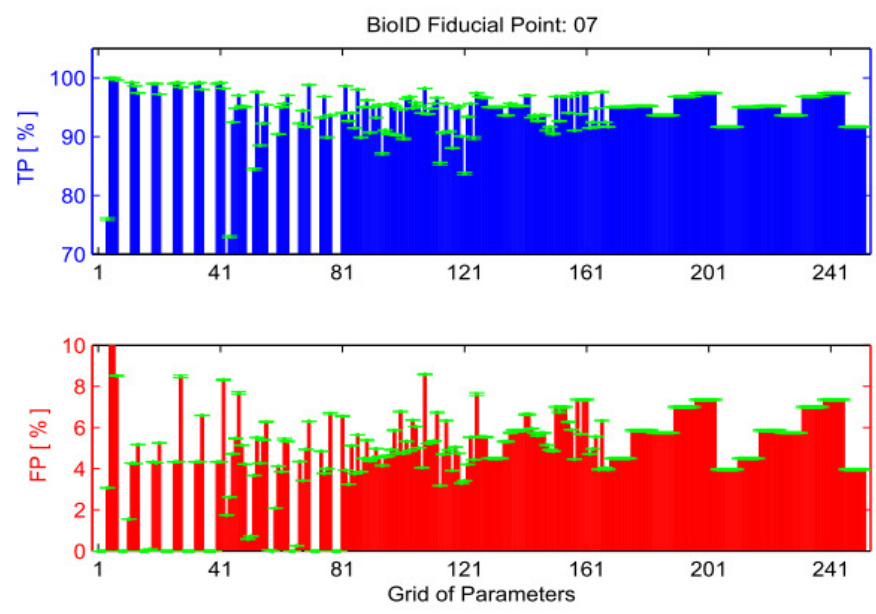

Figure 6. Grid of parameters for the point 07 (tip of nose) from BioID dataset.
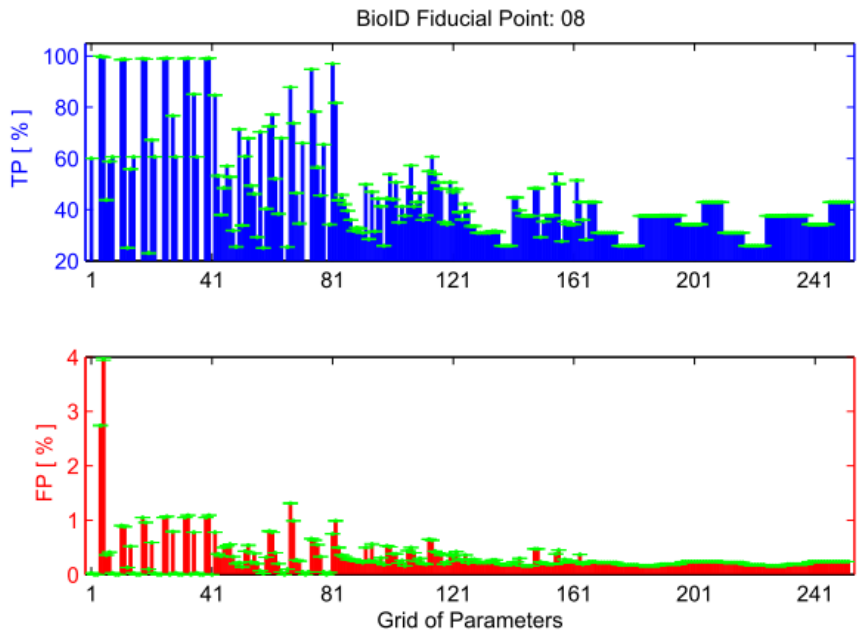

Figure 7. Grid of parameters for the point 08 (left nostril) from BioID dataset. 

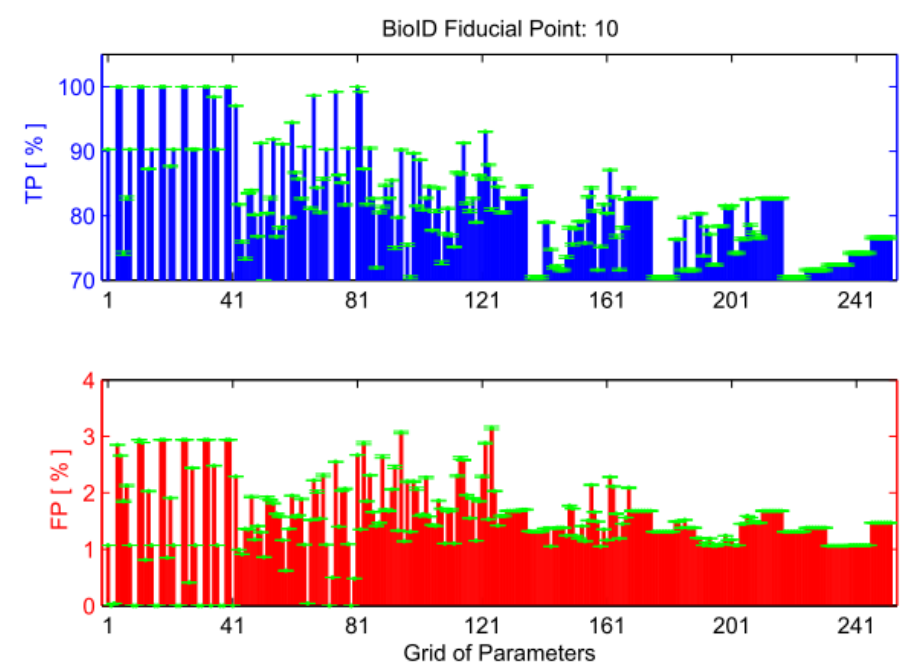

Figure 8. Grid of parameters for the point 10 (left mouth corner) from BioID dataset.

In Table I, we show the results of the proposed method with the best performance of parameters set for 11 fiducial points. The systems used in the methods SVM-L, DF and DF-PCA, are similar those presented in [1]. These results differ due to the method of post-processing. In our research, the post-processing is done by considering a region of $10 \%$ around the fiducial point for the calculation of true and false positives (TP and FP). We can see that, in Table I, the proposed method outperforms DF for all fiducial points, except in point 07 and outperforms linear SVM in points $0,1,2,4,5,9$ and 10. In points $0,1,3,4,5,8,9$ and 10, TP rate outperforms DF-PCA method. We consider FP rates less than $2 \%$ satisfactory, this fact can be observed in the proposed method, except in fiducial point 09.

Table I. Performance of the proposed system for 11 points using BioID database.

\begin{tabular}{|c|c|c|c|c|c|c|c|c|c|c|c|c|c|c|c|c|}
\hline \multirow[t]{2}{*}{ PF } & \multicolumn{4}{|c|}{ DF-PCA } & \multicolumn{4}{|c|}{ DF } & \multicolumn{4}{|c|}{ SVM L } & \multicolumn{4}{|c|}{ C-SVC (Proposed) } \\
\hline & FP & $\sigma$ & TP & $\sigma$ & FP & $\sigma$ & TP & $\sigma$ & FP & $\sigma$ & TP & $\sigma$ & FP & $\sigma$ & TP & $\sigma$ \\
\hline 0 & 0.05 & 0.03 & 96.6 & 2.2 & 0.22 & 0.03 & 21.1 & 4.7 & 1.09 & 0.74 & 92.4 & 9.8 & 0.76 & 0.07 & 99.2 & 0.74 \\
\hline 1 & 0.10 & 0.05 & 95.4 & 2.8 & 0.30 & 0.04 & 59.2 & 6.8 & 0.32 & 0.09 & 99.6 & 1.0 & 0.40 & 0.03 & 99.8 & 0.52 \\
\hline 2 & 0.24 & 0.14 & 95.0 & 2.1 & 0.39 & 0.03 & 53.7 & 6.1 & 1.58 & 0.59 & 93.0 & 14.3 & 0.76 & 0.17 & 92.3 & 4.2 \\
\hline 3 & 0.08 & 0.03 & 95.0 & 2.5 & 0.30 & 0.03 & 65.6 & 13.7 & 0.46 & 0.21 & 96.0 & 1.7 & 0.92 & 0.40 & 97.6 & 1.7 \\
\hline 4 & 0.11 & 0.04 & 97.8 & 2.8 & 0.40 & 0.02 & 65.0 & 9.6 & 2.49 & 4.76 & 97.2 & 4.2 & 0.50 & 0.11 & 99.9 & 0.67 \\
\hline 5 & 0.05 & 0.02 & 97.4 & 2.5 & 0.23 & 0.04 & 70.3 & 12.4 & 2.27 & 0.62 & 98.8 & 3.1 & 0.31 & 0.14 & 99.6 & 0.68 \\
\hline 6 & 0.14 & 0.03 & 93.2 & 1.6 & 0.25 & 0.04 & 56.7 & 9.3 & 1.40 & 1.44 & 99.6 & 1.0 & 1.95 & 0.79 & 90.5 & 11.74 \\
\hline 7 & 0.18 & 0.02 & 95.8 & 3.7 & 0.20 & 0.01 & 54.1 & 8.1 & 0.06 & 0.03 & 47.1 & 9.6 & 0.58 & 0.28 & 55.1 & 12.74 \\
\hline 8 & 0.17 & 0.02 & 94.8 & 2.6 & 0.23 & 0.01 & 54.9 & 5.0 & 0.40 & 0.09 & 95.4 & 3.9 & 0.75 & 0.15 & 97.0 & 2.32 \\
\hline 9 & 0.18 & 0.04 & 92.8 & 3.0 & 0.22 & 0.01 & 43.5 & 5.5 & 2.91 & 5.12 & 96.8 & 5.5 & 2.08 & 0.33 & 99.3 & 1.09 \\
\hline 10 & 0.18 & 0.02 & 87.4 & 2.9 & 0.11 & 0.04 & 22.1 & 6.2 & 1.47 & 0.49 & 98.6 & 1.9 & 1.34 & 0.50 & 99.2 & 0.74 \\
\hline
\end{tabular}

\section{CONCLUSION}

In this paper, we propose the performance evaluation of mathematical formulation SVM, called C-SVC employed in 11 fiducial points detection. In this research, we use parameters of C-SVC unexploited in the literature. The supervised system was implemented using the library in C++ called OpenCV. In this work, considering all the parameters of the system, we performed 8316 experiments. The performance of parameters grid in 5 fiducial points was shown and the best performance was compared with other similar systems in the literature. From this set, two classifiers were used for discriminating filter with and without PCA and one using a linear SVM. Evaluating the performance in terms of TP and FP rates using cross-validation with 7 folds, we identify the proposed system presents good results. Finally, we comment the relevance of this 
research in methods that provide fiducial points detection on human faces due to large number of applications.

\section{ACKNOWLEDGEMENT}

We would like to thanks Samsung for financial support. This research was supported by FAPEAM and CAPES agency.

\section{REFERENCES}

[1] W. S. da Silva Júnior, G. M. Araújo, E. A. B. da Silva and S. K. Goldenstein, "Facial Fiducial Points Detection Using Discriminative Filtering on Principal Components". In: Proceedings of the IEEE International Conference on Image Processing, September, pp. 2681-2684, 2010.

[2] S. Jahanbin, H. Choi and A.C. Bovik, "Passive Multimodal 2-D+3-D Face Recognition Using Gabor Features and Landmark Distances". IEEE Transactions on Information Forensics and Security, vol.6, pp.1287-1304, 2011.

[3] Du, Chunhua and Yang, Jie and Wu, Qiang and He, Xiangjian, "Locating Facial Landmarks by Support Vector Machine-based Active Shape Model". International Journal of Intelligent Systems Technologies and Application, vol. 10, n. 2, pp. 151-170, 2011.

[4] V. N. Vapnik, The Nature of Statistical Learning Theory. New York, NY, USA: Springer-Verlag New York, Inc., 1998.

[5] C. Chang and C. Lin, "LIBSVM: A Library for Support Vector Machines". ACM Transactions on Intelligent Systems and Technology,vol.2, n. 3, 2011.

[6] K. Wu and S. Wang "Choosing the Kernel Parameters for Support Vector Machines by the Intercluster in the Feature Space". Journal on Pattern Recognition, vol. 42, pp. 710-717, 2009.

[7] "Bioid Database", [Last access in May of 2013]. [Online]. Available in: http://www.bioid.com/

[8] G. M. Araujo, W. S. da Silva Júnior, E. A. B. da Silva, and S. K. Goldenstein, "Facial Landmarks Detection based on Correlation Filter", In: Proceedings of the IEEE International Telecommunication Symposium, Manaus, AM, Brazil, October 2010.

[9] "Cognitec Systems", [Last access in May of 2013]. [Online]. Available in: http://www.cognitecsystems.de/

[10] A. Pradhan, "Support Vector Machine - A Survey". International Journal of Emerging technology and Advanced Engineering, vol. 2, pp. 82-85, 2012.

[11] Viola, P. and Jones, M., "Robust Real-Time Object Detection". International Journal of Computer Vision, vol. 57, n. 2, pp. 137-154, 2001.

[12] Xiaoyang, T. and Triggs, B., Enhanced Local Texture Feature Sets for Face Recognition Under Difficult Lighting Conditions. IEEE Transactions on Image Processing, vol. 19, n. 6, pp. 1635-1650, 2010.

[13] G. R., Arce, J. L., Paredes and J. Mullan, "Nonlinear Filtering for Image Analysis and Enhancement". In: A. L. Bovik (Ed.), Handbook of Image \& Video Processing, Academic Press, 2000.

[14] "Open Computer Vision Library - OpenCV", [Last access in May of 2013]. [Online]. Available in: http://opencv.org/

[15] Reeves, S., "A Cross-Validation Framework for Solving Image Restoration Problems". Journal of Visual Communication and Image Processing, vol. 3, pp. 433-445, 1992.

[16] Kohavi, R., "A Study of Cross-Validation and Bootstrap for Accuracy Estimation and Model Selection", In: Proceedings of the International Joint Conference on Artificial Intelligence, August, pp. 1137-1143, 1996. 


\section{Authors}

Luiz Eduardo S. e Silva received the B.S. degree in electrical engineering at the Federal University of Amazonas (UFAM), Manaus, AM, Brazil, in 2011. Currently, he is a M.Sc. student at the same University and he is working with computer vision. Support Vector Machine is a technique applied in his pattern recognition researches, which try to classify elements of interest. $\mathrm{He}$ is Professor of Nokia Foundation in Electrical Circuits e Telecommunications. Automatic Control, Digital Signal Processing, Machine Learning and Mathematical Morphology are also his interest areas.

Pedro Donadio de T. Júnior is a researcher at Federal University of Amazonas - Brazil. He received his Eletrical Engineer degree in 2009 at Federal University of Amazonas. Currently, he is a M.Sc. student at the same University and he is working with computer vision. Discriminative filtering is a technique applied in his pattern recognition researches, which try to improve the filters performance by parallel processing. Automatic Control and noise cancellation are also his interest areas.

Kenny V. Santos is a graduate student in Electrical Engineering from Federal University of Amazonas. He received his bacharel degree in Electrical Engineering at Federal University of Amazonas in 2008. Currently he works in the fields of computer vision and pattern recognition, where his main interest is applications of digital signal processing in pattern recognition.

Waldir S. S. Júnior received the B.S. degree in electrical engineering at the Federal University of Amazonas (UFAM), Manaus, AM, Brazil, in 2000, and the M.S. degree in electrical engineering at the Federal University of Rio de Janeiro (COPPE/UFRJ), Rio de Janeiro, RJ, Brazil, in 2004 and Ph.D. degree in electrical engineering at the Federal University of Rio de Janeiro (COPPE/UFRJ), Rio de Janeiro, RJ, Brazil, in 2010. Since 2006, he has been with the Federal University of Amazonas, as Full Professor. His research interests are in the fields of data compression, as well as in mather gtay
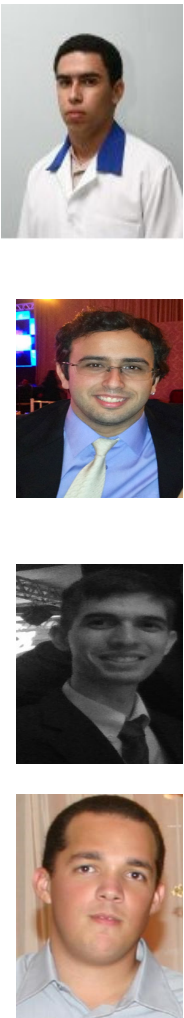